\title{
Synthesis of Selenium Nanoparticles using Sodium Selenite [Se(IV)] as A Precursor and Titanium(III) Chloride as A Reducing Agent
}

\author{
${ }^{1}$ Rajkumar Kalaparthi \\ Department of Engineering Chemistry, \\ A. U. College of Engineering, Andhra University, \\ Visakhapatnam - 530 003. Andhra Pradesh, India; \\ ${ }^{2}$ Srija Korapu \\ Department of Engineering Chemistry, \\ A. U. College of Engineering, Andhra University, \\ Visakhapatnam - 530 003. Andhra Pradesh, India;
}

\author{
${ }^{3}$ Himabindu Gandam \\ Department of Engineering Chemistry, \\ A. U. College of Engineering, Andhra University, \\ Visakhapatnam - 530 003. Andhra Pradesh, India; \\ ${ }^{4}$ Vijaya Raju Kurimella. \\ Department of Engineering Chemistry, \\ A. U. College of Engineering, Andhra University, \\ Visakhapatnam - 530 003. Andhra Pradesh India;
}

\begin{abstract}
A novel, simple, rapid and inexpensive wet chemical method has been developed to synthesis selenium nanoparticles by reduction of selenium (IV) using titanium(III)chloride as a reducing agent in acid medium at room temperature. The method is capable of producing selenium nano particles in a size ranging from $40-90 \mathrm{~nm}$ under ambient conditions. The synthesized nano particles have been characterized by UV-Visible Spectrophotometry, X-Ray Diffraction (XRD), Energy Dispersive X-Ray (EDS), Dynamic Light Scattering Particle Size Analyzer (DLS), Scanning Electron Microscope (SEM) and Transmission Electron Microscope (TEM) techniques. Crystalline selenium nanoparticles were obtained without post annealing treatment.
\end{abstract}

Keywords: Selenium Nanoparticles; titanium(III) as reductant; Synthesis and Characterisation

\section{INTRODUCTION}

Selenium is well known for its photoelectrical \& semiconductor ${ }^{1}$ properties and high reactivity towards a wealth of chemicals ${ }^{2}$,therefore, it finds numerous applications in several photoelectrical devices, solar cells, xerography $^{3}$ and in the production of functional materials ${ }^{2}$ such as $\mathrm{Ag}_{2} \mathrm{Se}, \mathrm{ZnSe}$, CdSe etc. However, the nanoselenium particles or fabricates are found to be associate with a large number of properties such as relatively low melting point, a high photoconductivity, high catalytic activity ( towards hydration and oxidation reactions), high refraction coefficient in devices and relatively large piezoelectric, thermoelectronic and linear optical responses ${ }^{4,5}$ compared to its bulk material. Hence, nanoselenium fabricates find broad applications in photoelectronic devices, photovoltaic cells, rectifiers, photographic exposure meters and xerography ${ }^{4,5}$. Colloidal/nano selenium has been employed in the preparation of nutritional suppliments ${ }^{6}$ and developed for applications in medical diagnostics ${ }^{7}$, and in some biological activities $^{8}$.

Thus, the synthesis and characterization of selenium nanoparticles have caused the greatest interest to researchers. A survey of literature reveals that the main nanofabrication methodologies adopted in preparing selenium nano particles are based on the chemical reduction of higher valent selenium to zero valent selenium using a suitable reductant based on 'bottom up approach'. In most of these methods selenium (IV) in the form of sodium selenite, selenous acid, or selenium dioxide (as a precursor) is reduced to elemental selenium in nano crystalline form employing various reducing agents such as dextrose ${ }^{9}$, sodium ascorbate ${ }^{10}$, ascorbic acid $^{11,12}$, hydrazine ${ }^{13,14}$, hydrazine hydrate (in the presence of polyvinyl pyrrolidine) ${ }^{15}$, sodium metabisulfite (in presence of sodium dodecylsulfate) ${ }^{16}$, sodium thiosulphate (in presence of surfactant stabilizer ${ }^{16}$, glutathione ${ }^{17}$, polyvinyl alcohol $^{18}$ etc. Other methods of producing nano selenium include $\gamma$-irradiation technique ${ }^{19}$, ultrasonic ${ }^{20}$ and laser ablation aproaches ${ }^{21}$, micelle mediated $^{22}$ and electro chemical synthesis techniques ${ }^{23}$, a fermentation procedure( in which probiotic lactic acid bacteria is made use of $)^{24}$ etc. The nanoparticles prepared with these methods are mostly spherical or quadrate in nature.

A careful delve into these methods reveals that most of them suffer from one disadvantage or the other. For example, the methods involving dextrose ${ }^{9}$, hydrazine $^{13,14}$, glutathione ${ }^{17}$ as reductants are tedious and time consuming while those with hydrazine hydrate ${ }^{15}$, sodium metabisulphite ${ }^{16}$ and sodium thiosulphate ${ }^{16}$ need the presence of some reagents (mentioned above in parenthesis) to stabilize the nano-selenium particles obtained. The reduction of selenium(IV) by sodium ascorbate $^{10}$ or ascorbic acid ${ }^{11,12}$ is extremely slow and requires about 5 hrs. to realize the formation of nano selenium. The reaction with polyvinyl alcohol ${ }^{18}$ must be carried out at elevated temperatures $\left(140^{\circ} \mathrm{C}\right.$ for $48 \mathrm{hrs}$.). The other types of methods cited above ${ }^{19-23}$, besides being tedious, necessitate use of the expensive state of the art technologies imperative and to provide appropriate culture medium for the growth of bacteria is a difficult task in the fermentation procedure ${ }^{24}$.

In the present paper, we demonstrated the use of titanium(III)chloride as a reducing agent to generate 
selenium nano particles and the procedure is free from the drawbacks of the earlier ones mentioned above. The method consists in treating aqueous solution of sodium selenite [selenium(IV)] taken as a precursor and IM hydrochloric acid medium with titanium(III)chloride solution [as a reductant] at room temperature. The method is simple, inexpensive, the reduction reaction is rapid and selenium nanoparticals of technologically useful range can be synthesized. Further, this method involves the use of all inorganic reagents, which are available in a high state of purity and hence can be easily and completely washed away from the selenium nanoparticle in the ambient aqueous medium by rising with distilled water. Moreover, the authors proposed the debut of titanium(III)chloride as a reducing agent for the synthesis of selenium nanoparticles.

\section{RESULTS AND DISCUSSION}

The reduction of sodium selenite or selenium(IV) to zero charged or elemental selenium with titanium(III)chloride in hydrochloric acid medium takes place according to the following chemical reactions.

$$
\begin{gathered}
4 \mathrm{Ti}(\mathrm{III}) \rightarrow 4 \mathrm{Ti}(\mathrm{IV})+4 \mathrm{e}^{-} \\
\mathrm{SeO}_{3}{ }^{2-}+6 \mathrm{H}^{+}+4 \mathrm{e}^{-} \rightarrow \mathrm{Se}+3 \mathrm{H}_{2} \mathrm{O} \\
4 \mathrm{Ti}(\mathrm{III})+\mathrm{SeO}_{3}{ }^{2-}+6 \mathrm{H}^{+} \rightarrow 4 \mathrm{Ti}(\mathrm{IV})+\mathrm{Se}(0)+3 \mathrm{H}_{2} \mathrm{O}
\end{gathered}
$$

titanium(III)chloride is only a mild reducing agent under normal experimental conditions.

A survey of literature revels that the reduction of selenium(IV) by various reductants were found feasible in hydrochloric acid medium or in presence of chloride ion ${ }^{30-}$ 32. Therefore, in the present investigation, selenium nano particles have been synthesized in the above acid medium containing chloride ion and in presence of a slight excess of titanium(III)chloride to ensure complete reduction of selenium(IV) by titanium(III)chloride.

\section{CHARACTERIZATIONS OF SELENIUM NANOPARTICLES}

The formation of selenium nanoparticles in presence of hydrochloric acid medium is primarily authenticated X-ray Diffraction Spectrum. The XRD pattern obtained from the as-prepared nanoparticles is shown in Figure 1. All the diffraction peaks in the XRD pattern could be indexed as the trigonal phase of selenium nanoparticles. All the peaks can be readily indexed to crystalline trigonal selenium (t-Se) (JCPDS 6-362) as a comparison. Although the diffraction peaks from XRD patterns of the raw selenium powders, can be well indexed to trigonal selenium with the P3121 space group, the strong diffraction intensity of the (101) peak is indicating the trigonal selenium(t-Se). Further, there is no clear change in peak positions as well as the absence of additional peaks indicate that the sample crystallizes in single-phase trigonal structure.

The average crystallite size of pure selenium nanoparticles was calculated using Debye Scherrer formula $^{33-35} \mathrm{~L}=0.92 \lambda /(\mathrm{B} \cos \theta)$ Where $\mathrm{L}$ is the crystallite size, $\lambda$, the $\mathrm{X}$-ray wavelength, $\theta$, the Bragg diffraction angle and $\beta$, the full width at half maximum (FWHM) and found to be about $65 \mathrm{~nm}$.

Figure 1. XRD Pattern for Selenium Nanoparticle

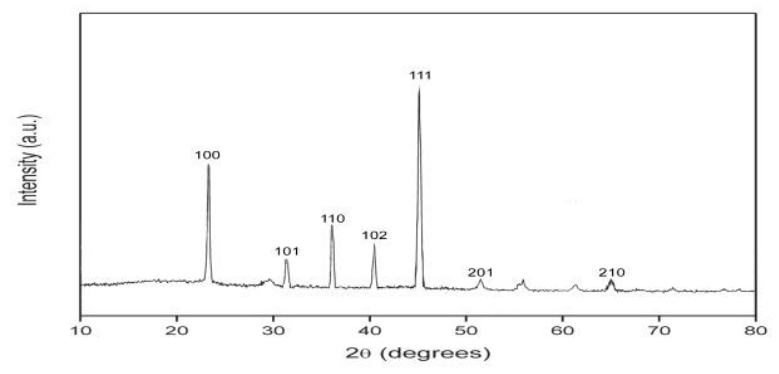

From UV-Vis spectra shown in Figure 2, it is observed that the $\lambda \max$ of the selenium nanoparticles was observed at $311 \mathrm{~nm}$ at lower concentrations where as in higher concentrations no specific $\lambda$ max could be identified rather a steady increase in absorbance from $800-300 \mathrm{~nm}$ had been observed. The UV-Visible spectra are in comparison to that of the selenium nanoparticles prepared by reducing with hydrazine hydrate ${ }^{36}$

Figure 2. UV-Visible Spectrum of the Se nanoparticles in solution

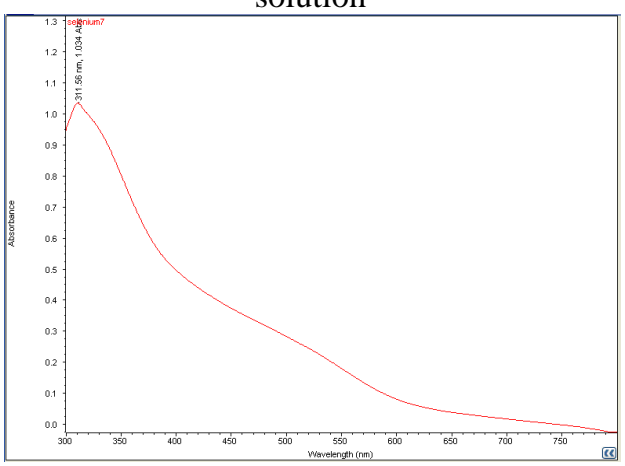

\section{FORM OF THE PRODUCED NANOPARTICLES - SEM, TEM AND EDX STUDIES:}

The formation of Selenium nanoparticles was verified by the corresponding SEM images presented in Figure 3. The selenium particles were found to coarse and irregular but well separated. It is well accepted that the formation of crystals is mainly achieved through two stages: nucleation and growth. As soon as the reaction starts, a certain amount of $\mathrm{t}$-Se seeds immediately precipitated out (i.e., nucleation) due to the reduced solubility of Se in the solution. The formation of these t-Se seeds relieved the level of super saturation and the following growth of residual a-Se colloidal particles present in the solution would occur on these t-Se seeds. Therefore, we reason that the strong dependence of the morphology of t-Se on the amount of Se in DEG solution should be ascribed to the variation in the amounts of both a-Se colloids and t-Se seeds. 
Figure 3. SEM images of selenium nanoparticles obtained using sodium selenite as a precursors and titanium(III) chloride as a reducing agent in $2 \mathrm{M}$ hydrochloric acid medium.


Figure 4. TEM images of selenium nanoparticles obtained using sodium selenite as a precursors and titanium(III) chloride as a reducing agent in $2 \mathrm{M}$ hydrochloric acid medium
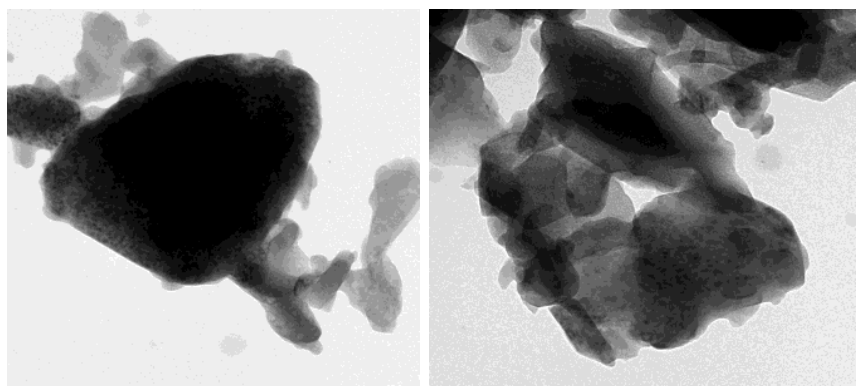

$\underline{100 \mathrm{~nm}}$

$\underline{100 \mathrm{~nm}}$
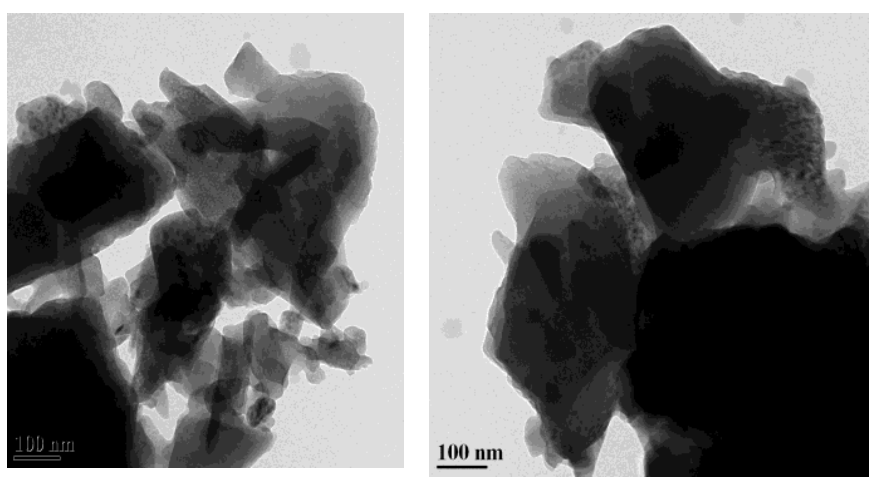

The TEM images shown in figure 4 reveal the agglomeration of the particles in solid phase where as in liquid phase there is a well separation of selenium nanoparticles.
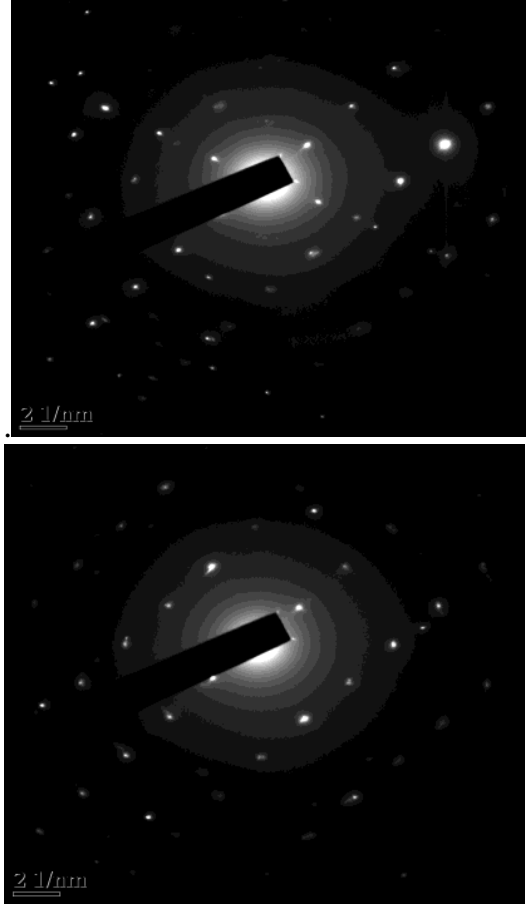

SAED pattern of tem images

The SAED images obtained from TEM studies clearly indicate that selenium nano particles are crystalline in nature. This is because the decrease in the viscosity of the medium $(2 \mathrm{M} \mathrm{HCl})$ allows the dissolution of amorphous selenium particles in the solution to be deposited on the few trigonal selenium seeds which are expected to be formed in the reaction medium.

The EDX spectra of the pure selenium nanoparticles is shown in Figure 5 and shows the peaks of pure selenium.

Figure 5. EDX image of Selenium Nanoparticles

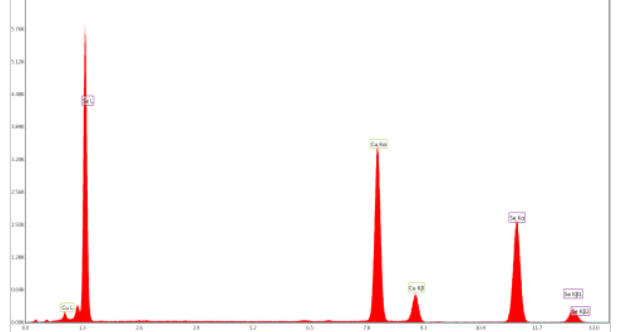

\section{Particle size Distribution:}

From Table 1 and Figure 6 the diameter histogram of the particle obtained from Light scattering particle size analysis in the solution form. Nearly $94 \%$ of the selenium particles were found to be below $100 \mathrm{~nm}$ in size and the average size of the particles was found to be around $60 \mathrm{~nm}$

Table 1. Particle Diameters and the \% frequency of the particles

\begin{tabular}{|c|c|c|}
\hline $\begin{array}{c}\text { Diameter } \\
(\mathrm{nm})\end{array}$ & $\begin{array}{c}\text { Frequency } \\
(\% / \mathrm{nm})\end{array}$ & Undersize (\%) \\
\hline 44.72 & 13.340 & 13.340 \\
\hline 50.53 & 21.019 & 34.359 \\
\hline 57.09 & 20.481 & 54.840 \\
\hline 64.50 & 16.159 & 70.999 \\
\hline 72.87 & 11.321 & 82.320 \\
\hline 82.33 & 7.346 & 89.666 \\
\hline 93.02 & 4.511 & 94.177 \\
\hline
\end{tabular}


Figure 6. Particle size distribution of Selenium

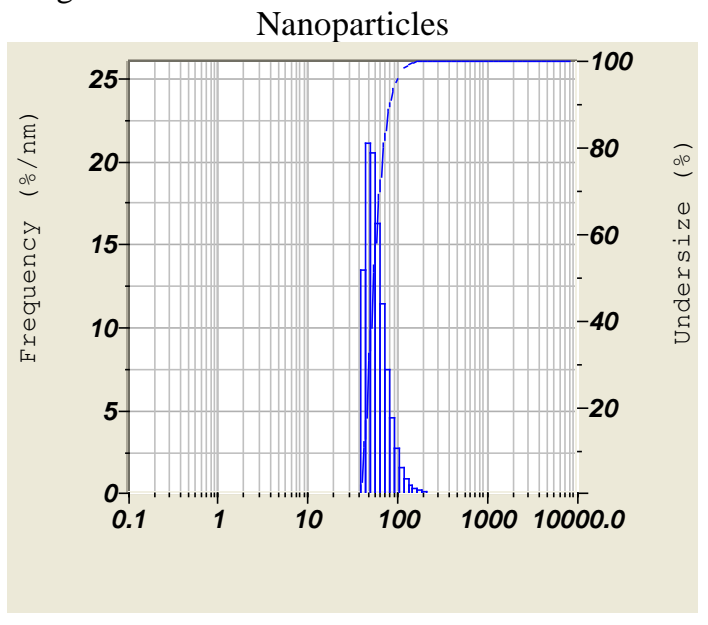

\section{EXPERIMENTAL SECTION}

All the reagents used of AR grade unless and otherwise stated and the solutions were prepared in double distilled water. A $0.25 \mathrm{M}$ solution of sodium selenite $\left[\mathrm{Na}_{2} \mathrm{SeO}_{3}\right]$ was prepared by dissolving the required quantity of the salt in distilled water in a $100 \mathrm{ml}$ standard flask. Simlarly a $0.75 \mathrm{M}$ solution of titanium(III)chloride was prepared from 30\%titanium(III)chloride solution in presence of hydrochloric acid medium in a $100 \mathrm{ml}$ standard flask.

A $10 \mathrm{ml}$ of $0.25 \mathrm{M}$ solution of sodium selenite was taken into a $250 \mathrm{ml}$ beaker. To this solution enough titanium(III)chloride and hydrochloric acids were added such that their overall concentrations are about $9 \mathrm{M}$ and $1 \mathrm{M}$ respectively when the solution is diluted to $100 \mathrm{ml}$. To this reaction mixture about $25 \mathrm{ml}$ of titanium(III)chloride solution of $0.75 \mathrm{M}$ was slowly added while it is being stirred mechanically on a magnetic stirrer. The stirring of the solution was continued for about ten minutes to ensure the complete reduction of selenium(IV) by titanium(III)chloride. The red viscous solution was then centrifuged; the precipitated selenium was collected, rinsed about five times with distilled water, followed by absolute ethanol, acetone and diethyl ether. The substance was then dried at about $40^{\circ} \mathrm{C}$ for two hours and taken for analysis.

Spectral studies were made using a ThermoScientific Evolution 201 Model UV-Visible Spectrophotometer. XRD patterns were obtained on a Philips X'pert MPD X-ray diffractomer using $\mathrm{Cu} \mathrm{Ka}$ $(1.054059 \mathrm{~A})$ radiation with the $\mathrm{X}$-ray generator operating at $45 \mathrm{KV}$ and $40 \mathrm{~mA}$. SEM images were obtained on JEOL 2010 microscopes. The particle image measurements were conducted on a Hitachi $\mathrm{H}-700 \mathrm{H}$ transmission electron microscope (TEM) operated at $150 \mathrm{kV}$ accelerating voltage. TEM samples containing nano-selenium particles were prepared by dip coating of the dispersed colloidal solutions on formvar/carbon film $\mathrm{Cu}$ grids (200 mesh; 3 mm, Agar Scientific Ltd.). Energy dispersive X-ray (EDS) analyses have also been conducted on the sample particles to verify their composition and purity. The resulting dispersions of selenium nanoparticles at various growth stages were subjected to the measurements for their structural images. It reflects the variation of particle mean diameters over a period of time due to agglomeration.

\section{CONCLUSIONS}

In summery, we have provided a convenient and fast approach for the preparation of selenium nanoparticles by reducing sodium selenite (Se IV) using titanium(III)chloride as a reductant in $2 \mathrm{M}$ hydrochloric acid medium. This method is simple and inexpensive for producing selenium nanoparticles for various applications.

\section{ACKNOWLEDGMENTS}

The authors are grateful to the authorities of Andhra University for the research facilities provided for undertaking the work and Dr.B.Kishore Babu for the work done on sem and tem images of selenium nanoparticles. Further, the authors wish to thank the Authorities of department of engineering chemistry A. U. College of Engineering (A), Andhra University for carryout this research.

\section{REFERENCES}

[1] Lide, D. V, 2002, Handbook of Chemistry and Physics, $83^{\text {rd }}$ ed., CRC Press, Cleveland, (Chapter 12)

[2] Gates, B.. Wu, Y.Y.; Yin, Y.D.; Yang, P. D.; Xia, Y. N. 2001, "Single - Crystalline Nanowires of $\mathrm{Ag}_{2} \mathrm{Se}$ Can be synthesised by Templating against Nanowires of Trigonal Se, J.Am.Chem.Soc., 123, pp.11500.

[3] Gates, B.; Mayers, B.; Cattle, B.; Xia, Y.N. 2002, "Synthesis and characterization of uniform nanowires of trigonal selenium" Adv.Funct.Mater. 12, pp.219.

[4] Berger, L. I. 1997; "Semiconductor Materials"; CRC Press; Boca Raton, FL, pp. 86-88.

[5] Johnson, J. A.; Saboungi, M. L.; Thiyagarajan, P. Csencsitn,R.; Meisel, D. "Selenium Nanoparticles: A Small Angle Neutron Diffraction Study", 1999, J. Phys. Chem. B, 103, pp.59-63.

[6] Yue-Hwa Chena, Hsiao-Pei Changa, Zong-Hong Linb and C R. Chris Wangb 2005, "Size effect of Colloidal Selenium Particles on the Inhibition of LPS-Induced Nitric Oxide Production", J. Chin. Chem. Soc., 52, 3, pp.389-393

[7] D. A. Yost, J. C. Russel, H. Yang, 1990, Non-Metal Colloidal Particle immunoassay, U.S. Patent 4, 954, 452 September 4,

[8] Peng.D; Zhang J, Liu Q, Taylor E.W. 2007, "Size effect of elemental selenium at nano size (Nano-Se) and supranutritional levels on selenium accumulation and glutathione $\mathrm{S}$ transferase activity" J. Inorg. Biochem 101, pp.1457-63

[9] Zong-Hong Lin, Fu-Chu Lin and C. R. Chris Wang, 2004 "Observation in the Growth of Selenium Nanoparticles", J. Chin. Chem. Soc. 51, 2, pp.239-242

[10] Mees, D. R., Pysto W., Tarcha, P. J. 1995, "Formation of Selenium Colloids using sodium ascorbate as the reducing agent", J. Colloid Interface Sci., 170, pp.254-260

[11] Sekhar, A. M. J., 1996, "Kinetics of the Reduction of Se(IV) to Se-Sol” J. Colloid Interface Sci. 180, pp.225-231

[12] Kumaran, C. K. S., Agilan, S., Velauthapillai, D., Muthukumaraswamy, N., Thambidurai, M., Senthil T. S. and Balasundaraprabhu, R., 2011, "Synthesis and Characterisation of Selenium Nanowires" International Scholarly Research Network, ISRN Nanotechnology, Article ID 589073

[13] B. Gates, Y. Yin, Y. Xia, 2000, "A Solution-Phase Approach to the Synthesis of Uniform Nanowires of Crystalline Selenium with Lateral Dimensions in the Range of 10-30 nm" J. Am.Chem.Soc. 122, pp.12582

[14] B.Gates, B. Mayers, B.Cattle, Y.Xia, 2002, "Synthesis and Characterization of Uniform Nanowires of Trigonal Selenium" Adv. Funct. Mater. 12, 3, pp.219-227

[15] Ji-Ming Song, Jian-Hua Zhu, and Shu-Hong Yu, "Crystallization and Shape Evolution of Single Crystalline 
Selenium Nanorods at Liquid-Liquid Interface: From Monodisperse Amorphous Se Nanospheres toward $\mathrm{Se}$ Nanorods" 2006, J. Phys. Chem. B110, 23790- 23795

[16] Zong-Hong Lin, C.R. Chris Wang, 2005, "Evidence on the size-dependent absorption spectral evolution of selenium nanoparticles" Materials Chemistry and Physics. 92, 2-3, pp.591-594

[17] Zhang, J., Wang, H, Bao Y, Zhang, Y., 2004, "Nano red elemental selenium has no size effect in the induction of seleno-enzymes in both cultured cells and mice" Life Sci. 75, pp. 237

[18] Shenglin Xiong. Baojuan Xi, Weizhi Wang, Chengming Wang, Linfeng Fei, Hongyang Zhou and Yiai Qian, 2006, Crystal Growth \& Design, 6(7), pp.1711-1716

[19] Y.J.Zhu, Y.T.Qian, H. Huang, M.W.Zhang, 1996, "Preparation of Nanometer size selenium powders of uniform size by $\gamma$ irradiation" Mater, Lett. 28, pp.119-122

[20] Li, X, M. ; Li, Y.; Li, Q.;Zhou, W.W.;Chu, H.B.; Chen. W.; Li, I.; Tang,Z.K. 2005, "Single Crystalline Trigonal Selenium Nanotubes and Nanowires Synthesized by Sonochemical Process" Cryst. Growth Des. 5, pp.911

[21] Jiang, Z. Y.; Xie, Z H. Xie, S. U; Zhang, X H, Huang, R.B.; Zheng, L. S. 2003, "High purity trigonal selenium nanorods growth via laser ablation under controlled temperature Chem.Phys. Lett. 368, pp.425-429

[22] Ma,Y, Qi, L. Ma, J. And Cheng, H. 2004, "Micelle Mediated Synthesis of Single crystalline selenium nanotubes" Adv. Mater, 16, pp.1023-1026

[23] Zhang, S.Y.; Liu, Y.; Ma, X.; Chen, H.Y. 2006, "Rapid Largescale synthesis and electrochemical behaviour of faceted single crystalline selenium nanotubes“" J.Phys. Chem. B 110, pp.9041-9047

[24] Peter Eszenyi, Attila Sztrik, Beata, and Joszef Prokisch, 2011, International Journal of Bioscience and Bioinfarmatics, 1(2), pp. $148-152$

[25] Gopal Rao, G. and Sagi, S.R., 1962, “A New Reductimetric Reagent : Iron(II) in a Strong Phosphoric Acid Medium Titration of Uranium(VI) with Iron(II) at Room Temperature" Talanta, ,.9, pp. 715

[26] John A. Dean, 1978, Lange's Hand Book of Chemistry, Mc Graw-Hill Book Company, $12^{\text {th }}$ Edition, pp. 6-17

[27] Vijaya Raju, K. and Madhu Gautam, G., 1988, Iron(II) Titration of Some Metal Ions , With Oxazine Dyes as Redox Indicators Talanta, 35(6), pp. 490-492

[28] Vijaya Raju, K. Rajasekhar Yadav, M. and David Sudhakar, G., 1994, "Iron(II) Titration of Some Quinones With Oxazine Dyes as Redox Indicators" Pro.Nat.Acad.Sci.India, 64(A), IV, pp 457

[29] Dikshitulu, L.S.A., Vijaya Raju, K. and Satyanarayana, D. 1977, Photometric Titration of Se(IV) with Iron(II)" Indian J. Chem 15A (4), pp 364

[30] Scherhaufer, A.M., 1958,“Zur Bestimmung des Selens mit Eisen (II)-sulfat" Fresenius Zeitschrift für Analytische Chemie 164 , pp 327-335

[31] Rooks, M. E.; Deshmukh, G. S.; Cook, E. R.; Sant, B. R.1952."Notes" Analyst, 77, pp 272-275

[32] Lingane, J.J. and Niedrach, L., 1948, "Polarography of selenium and tellurium; the -2 states" J.Am.Chem.Soc., 70(12), pp 4115-4120.

[33] Scherrer, P, 1918, "Bestimmung der Grösse und der Inneren Struktur von Kolloidteilchen Mittels Röntgenstrahlen, Nachrichten von der Gesellschaft der Wissenschaften, Göttingen," Mathematisch-Physikalische Klasse, Vol. 2, , pp. 98-100

[34] J. Langford and A. Wilson, "Scherrer after Sixty Years: A Survey and Some New Results in the Determination of Crystallite Size", Journal of Applied Crystallography, Vol. 11, 1978, pp. 102-103.

[35] Jenkins R and Snyder R L 1996 Introduction to X-ray powder diffractometry (John Wiley \& Sons Inc) pp 89-91

[36] Mehta, S. K., Savita Chaudhary and Bhasin K. K., 2009, "Understanding the role of hexadecyltrimethylammonium bromide in the preparation of selenium nanoparticles: a spectroscopic approach: J Nanopart Res 11, pp 1759-1766. 